PAPER • OPEN ACCESS

High harmonic generation in mixed XUV and NIR fields at a free-electron laser

To cite this article: Jan Troß et al 2022 J. Opt. 24025502

View the article online for updates and enhancements.
You may also like

\begin{tabular}{l} 
- Probing vortex beams based on Talbot \\
effect with two overlapping gratings \\
Sitti Buathong, Sorakrai Srisuphaphon and \\
Sarayut Deachapunya \\
- Thermodynamic approach for the \\
\hline understanding of the kinetics of heat eects \\
\hline induced by structural relaxation of metallic \\
Alasses \\
Alexandey Makarov, Gennadii V Afonin, \\
- Active rheology in odd viscosity systems \\
Cynthia J. Reichhardt and Charles \\
Reichhardt
\end{tabular}




\title{
High harmonic generation in mixed XUV and NIR fields at a free-electron laser
}

\author{
Jan Tro ${ }^{1}$, Shashank Pathak ${ }^{1}$, Adam Summers ${ }^{1}$, Dimitrios Rompotis ${ }^{2,3}$ (D), \\ Benjamin Erk $^{2}$ (D), Christopher Passow ${ }^{2}$, Bastian Manschwetus ${ }^{2}$ (D), Rebecca Boll ${ }^{3}$ (D), \\ Patrik Grychtol $^{3}$ (D), Sadia Bari ${ }^{2}$, Vinod Kumarappan ${ }^{1}$, Anh-Thu Le ${ }^{1,4}$, Cheng Jin $^{5}$, \\ Carlos Trallero ${ }^{6}$ (iD) and Daniel Rolles ${ }^{1, *}$ (i) \\ ${ }^{1}$ James R. Macdonald Laboratory, Department of Physics, Kansas State University, Manhattan, KS, \\ United States of America \\ ${ }^{2}$ Deutsches Elektronen-Synchrotron DESY, Hamburg, Germany \\ ${ }^{3}$ European XFEL, Schenefeld, Germany \\ ${ }^{4}$ Department of Physics, Missouri University of Science and Technology, Rolla, MO, \\ United States of America \\ ${ }^{5}$ Department of Applied Physics, Nanjing University of Science and Technology, Nanjing 210094, \\ People's Republic of China \\ ${ }^{6}$ Department of Physics, University of Connecticut, Storrs, CT, United States of America \\ E-mail: rolles@phys.ksu.edu
}

Received 14 August 2021, revised 1 December 2021

Accepted for publication 14 December 2021

Published 7 January 2022

\begin{abstract}
We present the results of an experiment investigating the generation of high-order harmonics by a femtosecond near-infrared (NIR) laser pulse in the presence of an extreme ultraviolet (XUV) field provided by a free-electron laser (FEL), a process referred to as XUV-assisted high-order harmonic generation (HHG). Our experimental findings show that the XUV field can lead to a small enhancement in the harmonic yield when the XUV and NIR pulses overlap in time, while a strong decrease of the HHG yield and a red shift of the HHG spectrum is observed when the XUV precedes the NIR pulse. The latter observations are in qualitative agreement with model calculations that consider the effect of a decreased number of neutral emitters but are at odds with the predicted effect of the correspondingly increased ionization fraction on the phase matching. Our study demonstrates the technical feasibility of XUV-assisted HHG experiments at FELs, which may provide new avenues to investigate correlation-driven electron dynamics as well as novel ways to study and control propagation effects and phase matching in HHG.
\end{abstract}

Keywords: high harmonic generation, free-electron laser, XUV-assisted HHG

(Some figures may appear in colour only in the online journal)

* Author to whom any correspondence should be addressed. 


\section{Introduction}

Driven by several technological break-through that led to the development of powerful x-ray free-electron lasers (XFELs) [1] and versatile high-order harmonic generation (HHG) sources [2-4], the last two decades have witnessed the emergence and rapid maturing of the new field of ultrafast $\mathrm{X}$-ray science $[5,6]$. However, despite considerable overlap between the communities involved in HHG and XFEL science and despite the potential for increased experimental flexibility and capabilities if both technologies were combined, only one experiment combining HHG and XFELs has been reported to date [7]. Here we report on the realization of another experiment of that type, but focusing on harmonic generation in a two-color field rather than using the HHG and XFEL pulses for a pump and probe experiment.

A number of years ago, it was shown theoretically that by combining an attosecond XUV pulse train with a nearinfrared (NIR) laser field, it is possible to achieve a dramatic enhancement of the HHG yield in a single atom or molecule [8-10] as well as in a macroscopic ensemble of atoms [11, 12]. Soon after these calculations were published, some of their predictions were experimentally verified, demonstrating an enhancement of the harmonic yield by a factor of five when the XUV field was present-even without phase control between XUV and NIR pulses [13, 14]. This effect was coined XUVassisted HHG, and following these initial calculations and experiments, a number of ideas for different 'flavors' of XUV or X-ray assisted HHG were developed. For example, the production of high harmonics in mixed fields at NIR intensities below the tunnel ionization threshold was observed [15], and it was shown that 'below-threshold harmonics' could be used to boost the HHG yield by altering the ionization step and thus increasing the conversion efficiency [16]. Several theoretical studies discussed how the HHG spectrum changes when a near-resonant XUV field produces a meta-stable excited electronic state that contributes to the HHG generation along with the ground state $[17,18]$. In particular, it was suggested that HHG radiation produced by a combination of an XUV pulse and an NIR field could be used to study correlationdriven dynamics such as Auger decay or correlation-driven inner-valence hole dynamics in excited atomic or molecular systems [19].

For most of these proposed applications, XUV pulse trains or single XUV pulses with pulse durations of a few femtoseconds or less, as well as a control of the delay between the XUV and NIR pulses on a sub-cycle level are assumed. However, it was also shown theoretically that longer and not fully coherent free-electron laser (FEL) pulses without phaselocking with the NIR field could be used for XUV-assisted HHG [20-22]. In these studies, XUV and X-ray-assisted HHG in $\mathrm{Ne}$ and $\mathrm{Kr}$ atoms using an XUV or XFEL was investigated. In particular, Buth et al [20] explicitly considered conditions that are available at the FLASH facility in Hamburg. Under these conditions, a second plateau in the HHG spectrum extending to much higher harmonic orders was predicted as a result of electron recombination with a core hole created by the XUV FEL pulse acting simultaneously with the NIR laser pulse [20]. Up to now, most of these more recent schemes and predictions for XUV-assisted HHG have not been verified experimentally.

Here we show that by combining femtosecond NIR pulses with XUV pulses from a FEL, the HHG yield can be suppressed or enhanced, depending on the FEL and laser intensities and on the timing between XUV and NIR pulses. Although we did not observe the clear and unambiguous effects predicted by theory, such as an up-shift of the harmonic cutoff by the photon energy, our results demonstrate the technical feasibility of XUV-assisted HHG experiments at a FEL. However, further and more systematic studies are needed to fully characterize the observed effects and to move beyond the proof-ofprinciple study reported here.

\section{Experimental setup}

The experiment was performed in the CAMP end-station [23] at beam line BL1 of the FLASH FEL facility at DESY in Hamburg, Germany [24]. FLASH was operated at a repetition rate of $10 \mathrm{~Hz}$ and an electron bunch charge of $0.23 \mathrm{nC}$, producing XUV pulses with a pulse energy up to $30 \mu \mathrm{J}$ on target at photon energies of 45.6, 82.6, and $95.3 \mathrm{eV}$, corresponding to XUV wavelengths of $27.2,15$, and $13 \mathrm{~nm}$, respectively. The latter two correspond to the $3 d \rightarrow 4 p$ resonance in singly ionized krypton, and to a photon energy just above the $\operatorname{Kr}(3 d)$ photoionization threshold, respectively. The XUV pulse durations were $\approx 50 \mathrm{fs}$ full width at half maximum (FWHM) at $27.2 \mathrm{~nm}$, $\approx 60 \mathrm{fs}(\mathrm{FWHM})$ at $13 \mathrm{~nm}$, and $\approx 85 \mathrm{fs}(\mathrm{FWHM})$ at $15 \mathrm{~nm}$, as determined from a measurement of the electron bunch duration $[25,26]$.

NIR pulses with a central wavelength of $810 \mathrm{~nm}$, a pulse duration of $60 \mathrm{fs}$ (FWHM in intensity), and a maximum pulse energy of $5 \mathrm{~mJ}$ were produced by a $10 \mathrm{~Hz} \mathrm{Ti}$ :Sapphire chirpedpulse-amplification laser system [27], which is synchronized to the master clock of the FLASH accelerator by an all-optical synchronization and timing stabilization system [28]. The precise timing between the NIR and XUV pulses was adjusted using a mechanical delay stage. By sorting the singleshot pump-probe data on the information recorded by a bunch arrival-time monitor (BAM) in the accelerator, the arrivaltime jitter between the XUV and NIR pulses can be partially corrected for and the overall temporal resolution can be improved to a point where the arrival-time jitter is smaller than the pulse durations of the NIR or XUV [29]. NIR and XUV pulses were both linearly polarized in the horizontal. The pulse energy (and, thus, peak intensity) of the laser field was adjusted with a computer controlled half-wave plate and polarizer. The pulse energy of the XUV beam was adjusted using two sets of metal filters installed upstream of the beam line optics. Unless noted otherwise, all values for the FEL pulse energy quoted throughout this manuscript refer to the FEL pulse energies after these filters, which were calculated from the pulse energies measured upstream of these filters using the filter transmissions obtained from calibration measurements [30]. We note that the actual filter transmissions may differ due to additional oxide layers that may have formed over time, which 


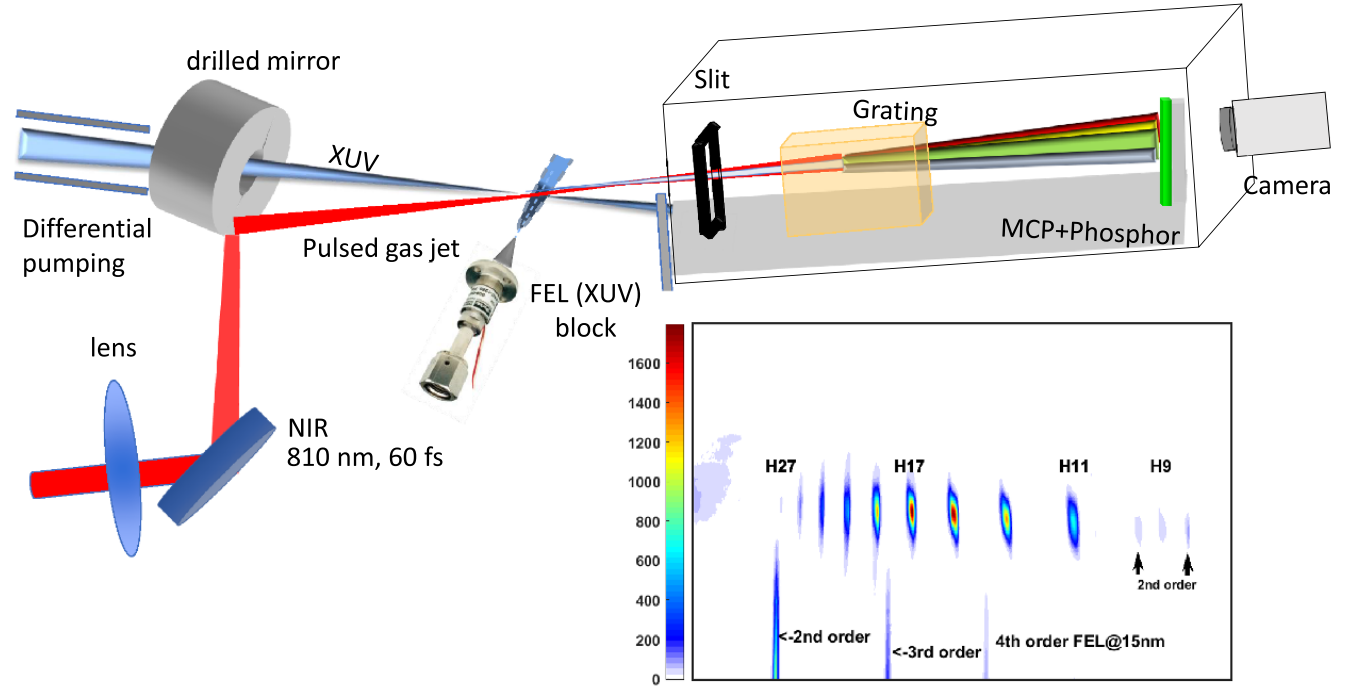

Figure 1. Sketch of the experimental setup. The NIR laser pulses are focused with a $f=75 \mathrm{~cm}$ lens and combined with the XUV pulses from the FEL in a near-collinear geometry using a drilled in-vacuum mirror. Close to the focus, a pulsed valve generates a dense gas target, in which higher harmonics are generated. A conical tip of the gas valve is used to reduce reflection of the NIR laser. The higher harmonics are frequency resolved in a home-built XUV spectrometer located downstream of the interaction region. A typical HHG spectrum generated in krypton is shown in the inset. The direct FEL beam is blocked by a beam block after the interaction region. However, stray light from the FEL can enter the spectrometer, and higher diffraction orders of this stray light are detected at the bottom end of the MCP detector.

generally decrease the transmission. Furthermore, additional transmission losses occur on the beam line transport and focusing optics. These losses have been characterized to some extent [23] and are also included in all given pulse energies.

The experimental setup for the generation and detection of the high harmonic signal (see figure 1) consisted of three separate vacuum chambers for NIR-XUV recombination, HHG production, and XUV spectroscopy, respectively, which were decoupled from the beam line vacuum by a window-less differential pumping unit. In the HHG production chamber, the NIR and XUV pulses were crossed with a dense beam of target gas produced by a pulsed Parker valve $(150 \mu \mathrm{m}$ nozzle diameter) operated at $10 \mathrm{~Hz}$ with a backing pressure of approximately $1.5 \mathrm{bar}$, placed close to the laser focus with a micrometer adjusted $x y z$-manipulator. In the recombination chamber upstream of the HHG chamber, the NIR beam, which was focused with a $f=75 \mathrm{~cm}$ lens outside of the vacuum, was overlapped with the XUV beam in a near-collinear geometry using a 1.5 -inch dielectric 45 -degree mirror with a $5 \mathrm{~mm}$ hole in the center. The XUV beam, which was focused by a pair of grazing-incidence Kirkpatrick-Baez mirrors, passed through the hole in the recombination mirror, while the NIR beam was placed next to the hole in order to avoid reflection losses and to maintain a clean beam profile. This slight off-axis reflection on the recombination mirror resulted in an angle of 1.3 degree between the NIR and the XUV beam. Apart from avoiding any clipping of the NIR beam on the hole in the mirror, the noncollinear geometry has the additional advantage that it separates the generated harmonics from the direct FEL beam, so that the latter can be dumped on a beam block before entering the XUV spectrometer. Furthermore, the small angle makes it possible to detect any angular dependence in the HHG generation due to the addition of the individual $\mathrm{k}$-vectors of the
XUV and NIR, which could alter the direction of the produced HHG emission. At the position of the gas jet, which was placed approximately $2-2.5 \mathrm{~mm}$ out of focus of the XUV beam in order to reduce multiphoton ionization by the strongly focused XUV and to produce an XUV beam spot that was comparable to the NIR beam spot, the XUV beam had a spot size of approximately $20 \mu \mathrm{m} \times 40 \mu \mathrm{m}$ (as determined from ray tracing [23]), while the NIR spot size at the focus of the NIR beam was measured to be $34 \mu \mathrm{m} \times 43 \mu \mathrm{m}$ (FWHM) by imaging the virtual focus created from the beam leakage through the final turning mirror on a CCD camera. The NIR focus was placed approximately $2 \mathrm{~mm}$ upstream of the center of the gas jet in order to optimize the harmonic brightness of short trajectories [31]. The peak intensities quoted for the NIR pulses throughout the manuscript are calculated based on the measured focus size given above.

The home-built XUV spectrometer, placed downstream of the generation chamber, consisted of an entrance slit with a fixed slit size of $300 \mu \mathrm{m}$, a laminar type XUV grating (Shimadzu 30-002) with a line spacing of 1200 lines $\mathrm{mm}^{-1}$, and a rectangular chevron-stack MCP and $\mathrm{P} 43$ phosphor screen detector. The visible-light image of the phosphor screen was recorded at $10 \mathrm{~Hz}$ by a CCD camera (Allied Vision Pike F-145B), which was synchronized to the FEL and NIR pulse arrival time. The camera images were recorded on a shot-by-shot basis along with all other relevant single-shot parameters such as the FEL and NIR pulse energy and the FEL bunch arrival time, and with slower-varying parameters such as the readings of all vacuum gauges.

For diagnostics purposes, a ion time-of-flight (TOF) spectrometer was integrated into the generation chamber, which was retracted during the HHG measurements but which could be moved into the interaction region for establishing the initial 
spatial and temporal overlap between NIR and XUV pulses and for (roughly) characterizing the NIR and XUV intensities by TOF mass spectrometry of rare gases.

In order to overlap the XUV and NIR pulses in space and time at the position of the gas jet, a multi-step procedure was used as described in detail in [32]. The XUV and NIR beams were first visually overlapped on a Ce:YAG-coated beam viewing screen that could be inserted into the interaction region and imaged via a long-distance microscope. Coarse temporal overlap between the XUV and NIR pulses was established using a fast photodiode installed between the interaction region and the XUV spectrometer to place the pulses to within approximately $20-50 \mathrm{ps}$ of each other. Finally, Xe gas was introduced in the chamber, and a step-like increase in the ratio of $\mathrm{Xe}^{3+}$ to $\mathrm{Xe}^{2+}$ ions, which were detected in the ion $\mathrm{ToF}$ spectrometer, was used to determine the temporal overlap to a precision better than the duration of the XUV and NIR pulses. Subsequent timing drifts were monitored using a streak camera and the BAM, as described in [29, 32]. Nevertheless, since the drifts could not be fully characterized and corrected for, we estimate the remaining uncertainty in the 'time zero' position during the HHG pump-probe scans to be approximately $100 \mathrm{fs}$

\section{Numerical simulations of the HHG process}

HHG in a single atom is typically described in the three-step model $[33,34]$, where after the initial (tunnel) ionization, the electron is accelerated in the electric field of the laser pulse and then returns to the parent ion when the electric field reverses its direction. It can recombine with the parent ion and emit its excess kinetic energy in form of a photon with a frequency $\omega$. The constructive interference over many cycles of the laser pulse leads to discrete frequencies $\omega$ that are odd multiples of the carrier frequency of the driving laser pulse. The quantitative rescattering (QRS) model can be used in order to quantify the yield of emitted photons [35].

When calculating the HHG yield from an extended medium, the phase of the emitted photons plays an important role. This originates from constructive and destructive interference of the emission from the individual atoms along the path through the medium and can alter the HHG yield significantly. In a first level approximation, considering the spatial extension of the gas medium only along the laser propagation direction and neglecting the absorption effect $[36,37]$, the yield of emitted photons in the $q$ th harmonic order can be calculated as:

$$
N_{\text {out }}^{q} \propto\left|D_{q}\right|^{2} L_{\text {med }}^{2} \operatorname{sinc}^{2}\left(\frac{\Delta k_{q} L_{m e d}}{2}\right),
$$

where $L_{m e d}$ is the length of the gas medium, $\Delta k_{q}$ is the phase mismatch between the fundamental laser field and the field of the $q$ th harmonic, and $D_{q}$ is the induced dipole at the $q$ th order, which is assumed to be a constant. The coherence length is defined as $L_{c o h}=\pi /\left|\Delta k_{q}\right|$, which defines the length over which the produced harmonics can be added coherently before any destructive interference occurs.
This phase mismatch $\Delta k_{q}$ for a given harmonic $q$ can be described as the sum of several contributions [38, 39],

$$
\Delta k_{q}=\Delta k_{\text {geo }}+\Delta k_{\text {neut }}+\Delta k_{\text {plas }}+\Delta k_{\text {atom }},
$$

in which $\Delta k_{\text {geo }}$ describes the geometric phase given by the Gouy phase; $\Delta k_{\text {neut }}$ is the neutral dispersion phase (which is positive); $\Delta k_{\text {plas }}$ is the plasma phase (which is negative); and $\Delta k_{\text {atom }}$ describes the intensity dependent atomic phase. $\Delta k_{\text {neut }}$ and $\Delta k_{\text {plas }}$ depend on the harmonic order $q$ and on the ionization fraction of the medium $\eta$, which is the key parameter in the present study:

$$
\Delta k_{q}=\Delta k_{\text {geo }}+\frac{q 2 \pi(1-\eta)}{\lambda} \Delta n-q \lambda P \eta N_{a t m} r_{e}+\Delta k_{\text {atom }} .
$$

Here, $\lambda$ is the fundamental wavelength of the driving laser, $P$ the gas pressure in atmospheres, $\Delta n=n_{\text {laser }}-n_{q}$ the difference in refractive index for the fundamental and the $q$ th order in the medium at the given pressure, $N_{\text {atm }}$ the gas density at $1 \mathrm{~atm}$, and $r_{e}$ the classical electron radius.

In order to model the results obtained from the present experiment, we have employed a more precise theoretical treatment of HHG in a gaseous medium based on the method described by Jin et al [40], in which the evolution of the spatiotemporal fields of the driving laser and the high harmonics are calculated numerically. Since the driving laser is much stronger than the high harmonics, Maxwell's equations for propagation of the driving field and the high-harmonic field in the gas jet are solved separately. In these equations, refraction and absorption by the neutral atoms as well as the free electron density depend on the gas pressure (or density). The source term, i.e. the induced-dipole, is calculated using the quantitative rescattering (QRS) theory [35]. Although it was shown in prior work that the full propagation model describes the HHG process more accurately, many of the effects we describe in the following can be discussed and rationalized in terms of the simplified equations presented above, which provide a more illustrative interpretation than the numerical model.

\subsection{HHG in combined XUV and NIR fields}

In order to simulate and understand the impact of the additional XUV beam on the high harmonic generation process in our experiment, we need to consider its influence on all given equations and processes. In the single atom picture, it will add an additional contribution that depends on the probability of ionization by the XUV and on the probability of the re-colliding photoelectron recombining with atomic ions created by the XUV. The latter depends strongly on the photoelectron kinetic energy, the ponderomotive potential of the laser field, and, in particular, also on the sub-cycle timing of the electron emission relative to the laser field, which is essentially what most previous XUV-assisted HHG experiments have studied. Furthermore, if the XUV has a significant probability to ionize the atom long before the maximum of the NIR field interacts with the atom, it is equivalent to say that the probability for tunnel ionization by the NIR field will decrease 
Table 1. Calculated XUV (single-photon) ionization probabilities of Ar and Kr as a function of FEL wavelengths and FEL pulse energies compared to the observed relative drop in the experimental HHG yield for harmonic 19 and 25. The ionization probabilities were calculated as the product of the ionization cross sections of all accessible electronic levels [42] and the photon fluence, assuming an elliptical beam spot of $20 \mu \mathrm{m} \times 40 \mu \mathrm{m}(\mathrm{FWHM})$ and beam line transmission of 0.56 at $27.2 \mathrm{~nm}, 0.42$ at $15 \mathrm{~nm}$, and $0.43 \mathrm{at} 13 \mathrm{~nm}$.

\begin{tabular}{|c|c|c|c|c|c|c|c|}
\hline $\begin{array}{l}\text { FEL } \\
\text { wavelength } \\
(\mathrm{nm})\end{array}$ & $\begin{array}{c}\text { Pulse } \\
\text { energy } \\
(\mu \mathrm{J})\end{array}$ & $\begin{array}{c}\text { Ar } \\
\text { ionization } \\
\text { probability }\end{array}$ & $\begin{array}{l}\text { Ar } \\
\text { exp. } \\
\text { H19 }\end{array}$ & $\begin{array}{c}\text { Ar } \\
\text { exp. } \\
\text { H25 }\end{array}$ & $\begin{array}{c}\mathrm{Kr} \\
\text { ionization } \\
\text { probability }\end{array}$ & $\begin{array}{l}\mathrm{Kr} \\
\text { exp. } \\
\mathrm{H} 19\end{array}$ & $\begin{array}{c}\mathrm{Kr} \\
\text { exp. } \\
\mathrm{H} 25\end{array}$ \\
\hline 27.2 & 22.4 & 0.266 & 0.258 & 0.37 & 0.646 & 0.387 & 0.394 \\
\hline 15.0 & 30.2 & 0.298 & 0.254 & 0.278 & 0.111 & 0.131 & 0.133 \\
\hline 15.0 & 10.5 & 0.103 & & & 0.038 & 0.050 & 0.058 \\
\hline 15.0 & 8.8 & 0.087 & 0.119 & 0.126 & 0.032 & 0.017 & 0.010 \\
\hline 13.0 & 8.6 & 0.069 & & & 0.066 & 0.094 & 0.090 \\
\hline 13.0 & 3.5 & 0.028 & & & 0.026 & 0.017 & 0.019 \\
\hline
\end{tabular}

since the effective ionization potential of the ion is larger than that of the neutral atom. And finally, the XUV pulse can also free an electron in a secondary ionization step after the initial tunnel ionization by the NIR field. If the two ionization events occur close in time, the first electron, which was released by the NIR field, can now recombine with a deeper (core) hole, emitting a higher energy photon [20].

First, we focus on the 'target depletion' effect due to ionization by the FEL, which is rather straightforward to model: When the XUV pulse precedes the NIR pulse, the XUV pulse can remove electrons from target atoms. The resulting ions have much higher ionization potentials, and their contribution to the HHG signals is therefore much smaller than that from neutral atoms since the strong-field ionization probability by the NIR (the first step in the HHG process) depends exponentially on the ionization potential [37]. This depletion of neutral atoms therefore results in an effective reduction of emitter atoms, which can be modelled by using a smaller 'effective' gas pressure in our numerical model.

According to our estimates of the ionization probabilities, which are presented in table 1 , the interaction of the XUV pulse with the target for the present experimental conditions is dominated by single-photon ionization. In the single-atom picture, the reduction of the HHG yield due to target depletion can be linearly scaled with the FEL pulse energy and with the photoionization cross section, as long as saturation effects and more complicated geometry effects (e.g. from not perfectly overlapping foci and/or from the non-uniform intensity and fluence profiles in both beams) are neglected.

In addition to changing the single-atom response, the XUVinitiated ionization also influences the phase matching and, thus, the overall HHG yield observed from a macroscopic medium such as the gas jet used in the present experiment. In particular, the FEL pulse will influence two terms in the phase matching equation, equation (3): the plasma-induced phase mismatch, and the neutral dispersion phase mismatch. In order to model the effect of the FEL pulse, we calculate the HHG spectrum for a $0.5 \mathrm{~mm}$ long Ar gas target placed downstream of the focus of an $810 \mathrm{~nm}, 59.4 \mathrm{fs}$ ( 22 cycles) NIR pulse with $4.0 \times 10^{14} \mathrm{~W} \mathrm{~cm}^{-2}$ (peak intensity at the focus) and a beam waist $w_{0}=40 \mu \mathrm{m}$. We assume that the HHG yield is collected within a cone of $2 \mathrm{mrad}$ in the far field. Given the parameters of the gas jet in comparison to other experiments [41], we expect the local Ar pressure to be around 50-100 torr. For comparison, we also performed the same calculations for an NIR peak intensity of $2.0 \times 10^{14} \mathrm{~W} \mathrm{~cm}^{-2}$ and found qualitatively similar results to the ones described below, albeit with a significantly reduced red shift when modeling the effect of the FEL. It is important to note, however, that our model calculations are performed for an idealized case of constant gas density and constant fluence of the FEL pulse throughout the interaction region, which is not consistent with the actual experimental conditions and which may lead to significant deviations, as we will discuss in the following along with the experimental results.

The effect of the additional ionization by the FEL can also be described by adding an additional term $\eta_{F E L}$ to the ionization fraction $\eta$ in equation (3), which can be controlled independently in the terms describing the neutral dispersion phase mismatch and the plasma-induced phase mismatch. Ionization by the FEL will therefore affect both the single-atom response in the sense that it reduces the overall HHG yield since less emitters are present, and it affects the phase matching, thus adding an additional dependence of the yield on the harmonic order.

\section{Results and discussion}

Typical HHG spectra produced by the NIR pulses in the presence of the XUV pulses from the FEL are shown in the bottom right of figure 1 and in figure 2(b). Our detector range starts at approximately $12.5 \mathrm{eV}$, allowing us to capture the emission of the 9th harmonic (H9), and ends at $240 \mathrm{eV}$. The harmonics are spaced by $3.1 \mathrm{eV}$, as expected given the wavelength of the NIR laser. The HHG spectrum has the typical shape consisting of 'plateau' harmonics and an exponentially decaying yield at the cutoff. For the given gas pressure and NIR intensity, the highest harmonic with a signal clearly above the noise level is the 27th harmonic (H27). The observed cutoff of the harmonics followed the expected intensity dependence, i.e. the observable number of harmonics was reduced when the peak intensity of the NIR pulse was reduced. The smeared-out signal at the left-hand side of the detector in figure 1 is independent of the FEL pulse energy and most likely stems from a reflection of high-harmonic light on the pulsed valve. We also 

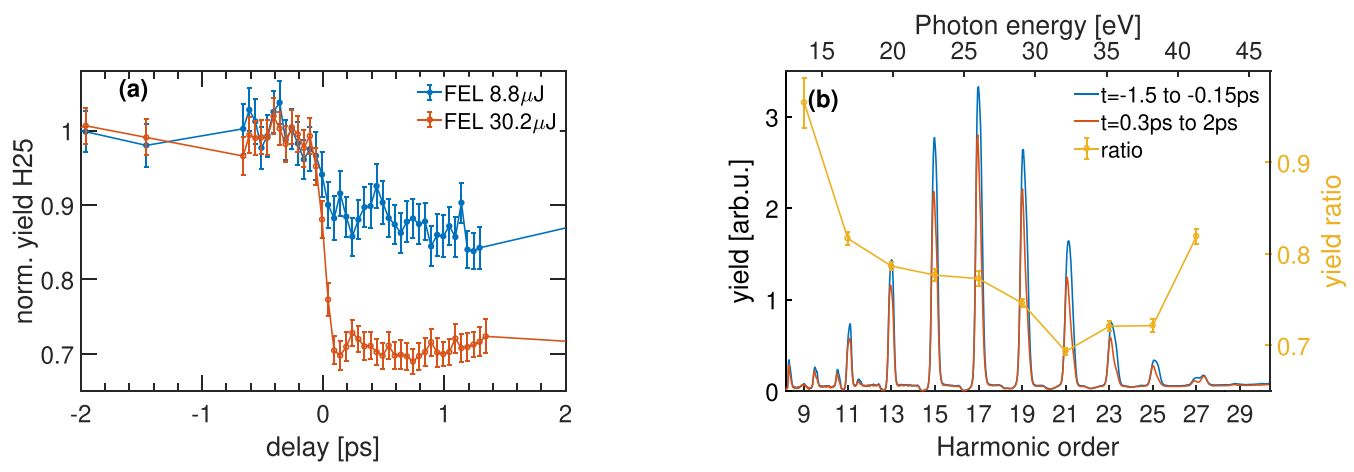

Figure 2. (a) Yield of the 25th harmonic of the fundamental NIR drive laser produced in argon as a function of delay between NIR and FEL pulses for two different FEL pulse energies $(8.8$ and $30.2 \mu \mathrm{J})$ and for an NIR field with a peak intensity of $2.7 \times 10^{14} \mathrm{~W} \mathrm{~cm}{ }^{-2}$. At positive delays, the FEL pulse, which has a photon energy of $82.6 \mathrm{eV}$, precedes the NIR pulse. The HHG yield at negative delays (NIR preceding FEL) was normalized to one. The error bars for the yield here and in all following figures represent the $2 \sigma$ error of the mean. (b) Averaged HHG spectrum in Ar produced by a NIR pulse with a peak intensity of $2.7 \times 10^{14} \mathrm{~W} \mathrm{~cm}^{-2}$ arriving between 1.5 and $0.15 \mathrm{ps}$ before the XUV pulse (blue), and between 0.3 and 2 ps after the XUV pulse (red), which has a pulse energy of $30.2 \mu \mathrm{J}$ at a photon energy of $82.6 \mathrm{eV}$. The yellow data points show the ratio of the integrated yield of each harmonic order for the two spectra shown in red and blue.

observe signal from higher-order diffraction in the detector plane. Specifically, the second orders of H17 and H19 on the left and right of the first diffraction order of H9 and diffraction orders 2, 3 and 4 of the FEL at $15 \mathrm{~nm}$ are visible in the detector image of figure 1 and in the spectrum in figure 2(b). The latter was produced by integrating over a region of interest at the center of the detector chosen to primarily include the HHG signal and to cut out most of the higher orders of the FEL beam.

\subsection{Delay-dependence of the HHG yield}

To investigate the dependence of the HHG yield on the delay between the XUV and the NIR pulses, we scanned the arrival time of the NIR pulses and collected delay-dependent data in multiple loops in order to minimize the effect of laser and FEL fluctuations. Additionally, a fast shutter in the FEL beam was used to collect NIR-only HHG spectra every 10th shot, which were used to monitor drifts, e.g. in the gas pressure, the NIR intensity, or the beam pointing. In the data analysis, we only considered shots where the FEL and NIR pulse energies were within $\pm 20 \%$ of the mean. Typically, this resulted in an acceptance rate of $50 \%-75 \%$ of the available events. For two data sets, recorded in argon at $15 \mathrm{~nm}$ with 30.2 and $8.8 \mu \mathrm{J}$ FEL pulse energy, a normalization of the harmonic yield on the gas pressure was performed since the gas pressure was unstable during those runs. As the gas pressure was slowly dropping by $6 \%$ over the length of the data set (for $30.2 \mu \mathrm{J}$ ), the harmonic yield of the recorded 'NIR-only' shots dropped by $10 \%$, and this drop was corrected for by normalizing on the linearly scaled recorded gas pressure (i.e. for the largest pressure drop of $6 \%$, the yield was multiplied by a factor of 1.1).

As an example for the observed delay dependence of the HHG yield, figure 2(a) shows the dependence on the yield of the 25th harmonic generated in Ar as a function of the delay between the XUV and the NIR pulses for two different
FEL pulse energies. At negative delays, the NIR pulse arrives before the FEL pulse, and high harmonics are produced from an unperturbed target. When the XUV and NIR pulses overlap in time, we observe a sharp drop in the HHG yield, and the reduction in yield persists for all delays where the XUV pulse precedes the NIR pulse. At an FEL pulse energy of $30.2 \mu \mathrm{J}$, corresponding to a peak intensity of the XUV field of $4.9 \times$ $10^{12} \mathrm{~W} \mathrm{~cm}^{-2}$ in the interaction region (assuming a beam line transmission of $42 \%$ at a photon energy of $82.6 \mathrm{eV}$ ), the yield at positive delays is reduced by $30 \%$ compared to negative delays, where the NIR interacts with the gas medium before the FEL pulse arrived. At an XUV pulse energy of $8.8 \mu \mathrm{J}$, corresponding to a peak intensity of $1.2 \times 10^{12} \mathrm{~W} \mathrm{~cm}^{-2}$, the drop in the HHG yield is only $12 \%$.

To investigate the decrease in the HHG yield in more detail, figure 2(b) shows the full HHG spectrum for two delay intervals before and after the FEL pulse arrived. While the drop in the yield is observed for all harmonic orders, the magnitude of the decrease varies significantly as a function of the harmonic order. Furthermore, the HHG spectrum recorded after the arrival of the FEL pulse (red line) is shifted to slightly lower photon energies as compared to the unperturbed spectrum (blue line). The delay dependence of this shift displays the same behavior as the HHG yield, with a large jump at zero delay and no statistically significant variation at larger delays before and after the time overlap.

By recording similar scans for different FEL pulse energies, XUV wavelengths, NIR laser intensities, and target gases, we find that the magnitude of the drop in the HHG yield and the energy shift of the HHG spectrum depends on all of the above parameters. Figures 3(a) and (b) show the magnitude of the drop for Ar and $\mathrm{Kr}$ at two different FEL wavelength as a function of harmonic order, while figures $3(\mathrm{~d})$ and (e) show the corresponding energy shift. In all cases, the decrease in the yield is relatively small for the harmonics close to threshold, reaches a maximum in the region of the harmonics with the highest overall yield, and then decreases again for the harmonics close to 


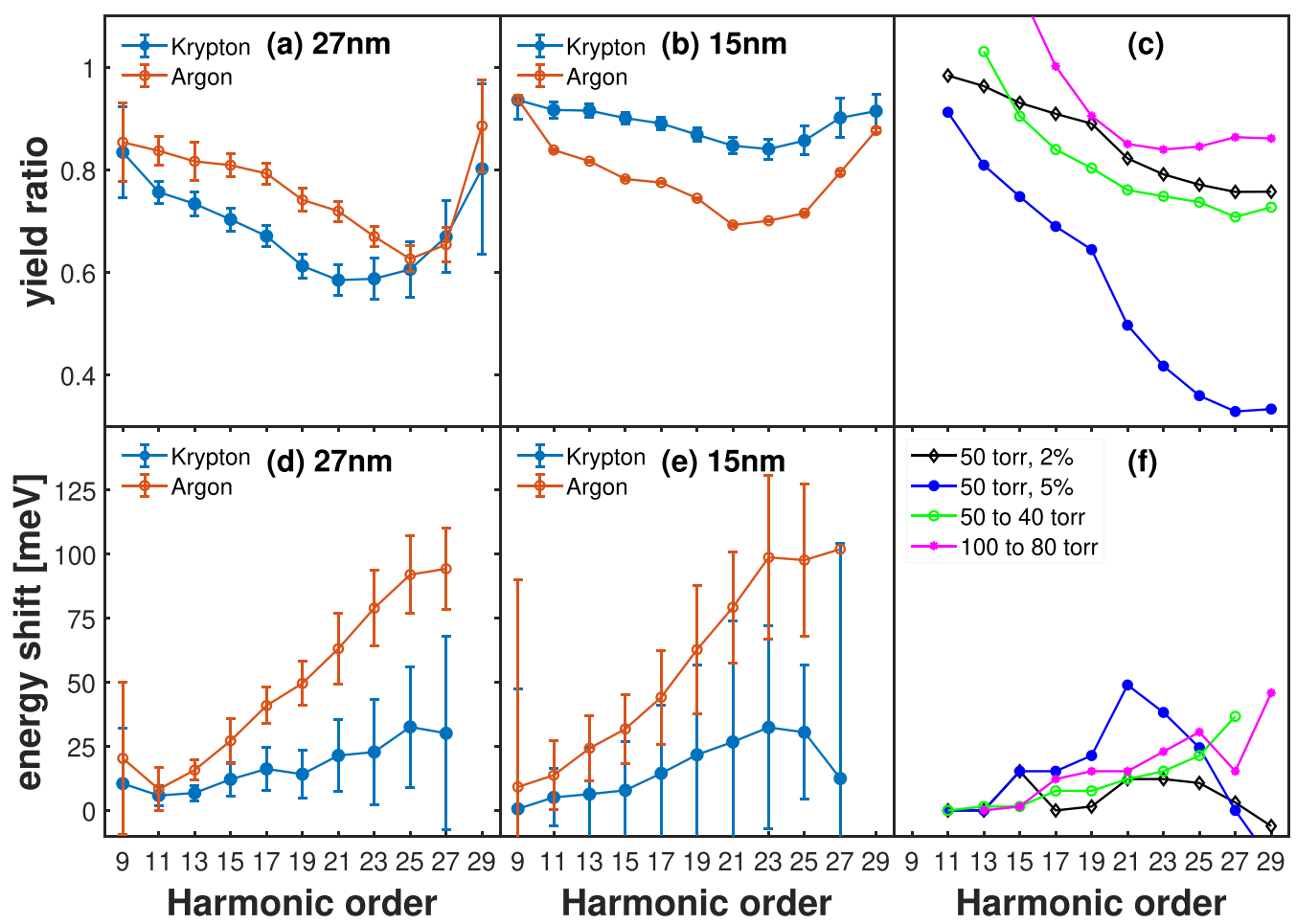

Figure 3. Ratio of the HHG yield before and after the drop in the time-dependent yield of various harmonics generated in argon and krypton (a) illuminated with XUV at $27.2 \mathrm{~nm}$ and $22 \mu \mathrm{J}$ pulse energy and a NIR peak intensity at $2.7 \times 10^{14} \mathrm{~W} \mathrm{~cm}^{-2}$; (b) illuminated with XUV at $15 \mathrm{~nm}$ and $30 \mu \mathrm{J}$ pulse energy and a NIR peak intensity of $3.2 \times 10^{14} \mathrm{~W} \mathrm{~cm}^{-2}$. (c) Theoretical calculations of the yield ratio in argon as a result of a reduced number of neutral atoms, modeled as a reduction in the gas pressure by $20 \%$ (green and magenta lines, see text) and for two different ionization fractions (2\% and 5\%, black and blue lines, respectively) at a fixed pressure. (d), (e) Difference in photon energy for discrete harmonic orders before and after the FEL interacts with the medium for the same conditions as in panels (a) and (b), respectively. (f) Theoretical calculations of the energy shift in argon for same conditions as in (c).

the cutoff, while the observed energy shifts increases steadily as a function of harmonic order.

\subsection{Dependence of the HHG suppression on FEL pulse energy and photon energy}

In order to estimate quantitatively what fraction of the target is ionized by the FEL pulse for a given set of FEL parameters, table 1 lists the calculated (single-photon) ionization probabilities of Ar and $\mathrm{Kr}$ for each FEL wavelength and pulse energy. Furthermore, the relative magnitude of the experimentally observed drop in the HHG yield for two harmonics, $\mathrm{H} 19$ and H25, is shown. The table shows a clear correlation between the ionization probability by the FEL and the magnitude of the drop in the HHG yield, with an approximately linear relationship except for the case with the highest ionization probability in $\mathrm{Kr}$, where the magnitude of the decrease seems to have reached saturation. These observations suggest that the dominant cause of the experimentally observed drop is the removal of neutral emitters in the target due to ionization by the FEL pulse, accompanied by a smaller effect due to phase matching that decreases the effective reduction for lower harmonic orders.

In order to test these hypotheses, we have performed model calculations for the case of Ar, shown in figures 3(c) and (f). To investigate the different contributions to the phase matching independently, our model calculations are done for different scenarios: First, we model the removal of $20 \%$ of the neutral atoms in the target due to ionization by the FEL pulse by reducing the partial pressure of the gas jet in our calculations from 50 to 40 torr (green line) and from 100 to 80 torr (magenta line). As described in section 3.1, this allows us to model the effect of a reduction of neutral atoms independent of the ionization fraction. In the simplified phase matching model described by equation (3), this changes the neutral dispersion phase by affecting $\Delta n$, which is a function of pressure, and the plasma phase, which explicitly depends on the pressure. Next, we model the effect of the plasma dispersion phase mismatch due to the additional electrons created by the FEL by increasing the ionization fraction $\eta$ in our calculations by $2 \%$ (black line) and 5\% (blue line) while keeping the partial pressure constant at 50 torr.

The results of the former model calculations qualitatively reproduce the harmonic-order-dependent drop in the yield and the red shift of the harmonics, although the latter is underestimated by the calculations. In particular, our model calculations demonstrate that the observed red shift is, indeed, better described as a reduction of the blue shift of the harmonic emission that occurs due to the reshaping of the electric field while propagating through the medium $[43,44]$. As the number of 
atoms that can be ionized by the NIR pulse is reduced, the harmonic spectrum is less blue shifted, thus resulting in a red shift when the spectrum is compared to the unperturbed emission spectrum before the FEL pulse has interacted with the target, as shown by the green and magenta lines in figure 3(f).

However, apart from this qualitative agreement regarding the general trends, there are some distinct differences between the experimental observations and the results of our model. Our calculations fail to reproduce the behavior for the cutoff harmonics (H27 and H29), for which the experimental data show a significantly smaller drop in yield than for the plateau harmonics, while this is not the case in the calculations. This might be caused by a non-perfect spatial overlap between the laser and the FEL focus. The cutoff harmonics are produced at the highest NIR intensities near the center of the laser focus, and if there is a slight mismatch between the two foci, the ionization due to the FEL may actually be slightly less in this region than the overall average.

Furthermore, our calculations significantly overestimate the decrease in the HHG yield due to the increased ionization fraction, even though the increase of the ionization fraction in our model calculations is chosen much smaller than what our estimates in table 1 suggest. At an ionization fraction of $20 \%-30 \%$, harmonic emission would be completely suppressed, which is clearly not the case in the experiment. This suggests that the calculations overestimate the change in the plasma dispersion phase mismatch due to the ionization by the FEL.

One explanation for this apparent contradiction could be that the electrons produced by the FEL contribute less to the plasma phase mismatch, e.g. if those electrons very quickly leave the region of space that contributes to the HHG generation. Alternatively, recombination of the photoelectrons with the surrounding ions in the plasma that is created by the interaction of the XUV pulse with the dense gas jet could result in a reduction of the density of free electrons and thus decrease the effect of the plasma phase mismatch. However, we do not observe any time dependence of the yield or red shift on a longer time scale which would support this hypothesis.

Even though we cannot pinpoint the exact cause of the disagreement, our observations lead us to the conclusion that our propagation model might not be suitable for describing a situation with a large ionization fraction and, thus, significantly overestimates the effect of the plasma phase mismatch. Indications for such a failure of existing propagation models were also found in experimental studies of HHG with a shorterwavelength driver in the ultraviolet, where a relatively high degree of ionization occurs and a significantly higher HHG yield was observed than expected based on the ionization fraction [45].

Another difference between the present experiment and all other experimental and theoretical investigations of the phase matching in HHG, which are reported to date, lies in the fact that the XUV pulses from the FEL are produced by selfamplified spontaneous emission and, therefore, are not fully temporally coherent and exhibit a spiky spectral and temporal shape. Although it is not immediately clear how this would affect any of the model predictions, it is important to keep this difference in mind.

Note that an additional complication in the quantitative comparison between experiment and model calculations may occur in case there is only partial overlap between the FEL and the NIR foci in the experiment such that the NIR effectively 'sees' a smaller ionization fraction than estimated in table 1 . In other words, as alluded to above, it is possible that the ionization probability of the FEL is not constant over the volume where the harmonics are generated, which will affect our model calculations of the macroscopic response. Furthermore, the overlap between FEL and NIR pulses may vary shot-to-shot due to small pointing fluctuations such that the experimental results are (possibly) averaged over a range of ionization fractions.

\subsection{Enhancement of the HHG yield in the presence of the XUV field}

In most of the delay scans we performed, we observe a simple step in the HHG yield centered at times close to 0 ps delay, as shown, e.g. in figure 2(a) and discussed in the previous sections. However, in all of the scans performed in krypton gas at an XUV photon energy of $82.6 \mathrm{eV}$, we consistently observe a small increase in the HHG yield starting approximately $2 \sigma$ (based on the pulse durations of XUV and NIR) or approximately $200 \mathrm{fs}$ earlier in time than the center of the step function describing the drop in HHG yield, i.e. when the NIR pulse overlaps with the rising edge of the XUV pulse. Figure 4 shows the corresponding delay dependence of the yield of harmonic 19 for three FEL intensities. As discussed in the previous sections, the magnitude in the observed drop depends on the FEL intensity and changes from a difference of $15 \%$ for the highest FEL intensity to a $2 \%-5 \%$ drop for a lower FEL intensities. The relative change has the same dependence on the harmonic order as shown in figure 2(b), independent of FEL intensity. In addition to the drop, a small enhancement of the HHG yield right before the drop is visible at the lowest FEL intensities. This enhancement of the HHG yield can be investigated more systematically by integrating the yield in the delay region between -200 and -50 fs and by plotting the enhancement as a function of harmonic order for the different FEL pulse energies. This is shown in figure 5(a), where the enhancement is defined as the average yield of each harmonic for delays between $-200 \mathrm{fs}$ and $-50 \mathrm{fs}$, normalized to the yield at very negative delays, when the NIR drives HHG from a target that was not yet illuminated by the FEL. For the highest FEL pulse energy, no increase in the harmonic yield is visible. When reducing the FEL pulse energy, a small but statistically significant enhancement appears that increases with decreasing FEL pulse energy and is largest for the highest harmonic orders detected. At the lowest pulse energy, we observe an enhancement of 3\%-6\%. Note that the data represented by blue circles and orange diamonds were taken at the same FEL pulse energy and only differ in that more statistics were taken for the former, which was only recorded at four delay points. The NIR intensity was set to $2.7 \times 10^{14} \mathrm{~W} \mathrm{~cm}^{-2}$ for all of these scans. 


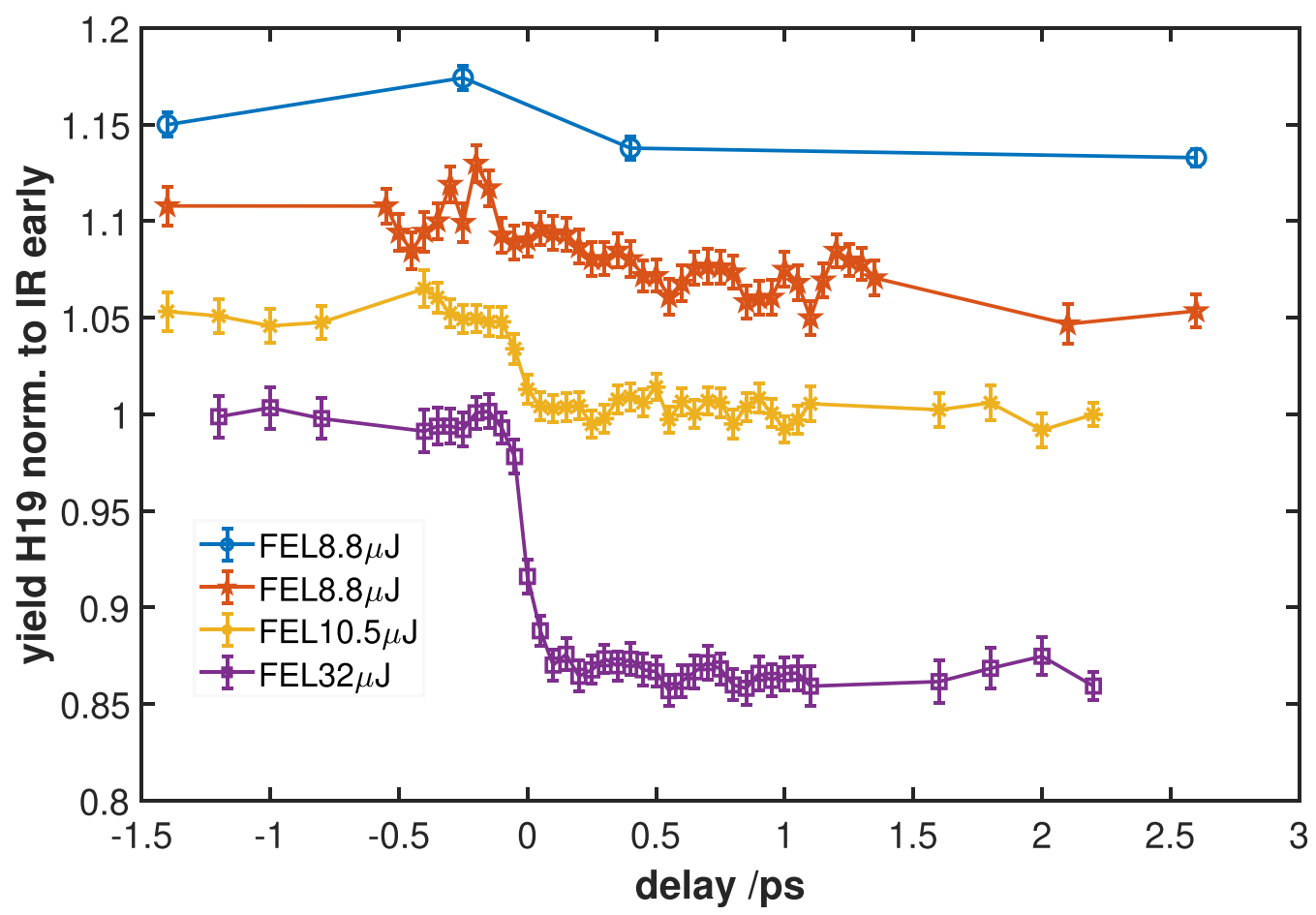

Figure 4. Yield of the 19th harmonic of the fundamental laser produced in $\mathrm{Kr}$ as a function of delay between NIR and FEL pulses for different FEL pulse energies at an FEL photon energy of $82.6 \mathrm{eV}$ and an NIR intensity of $2.7 \times 10^{14} \mathrm{~W} \mathrm{~cm}^{-2}$. Each line is vertically offset by 0.05 for better visualisation.

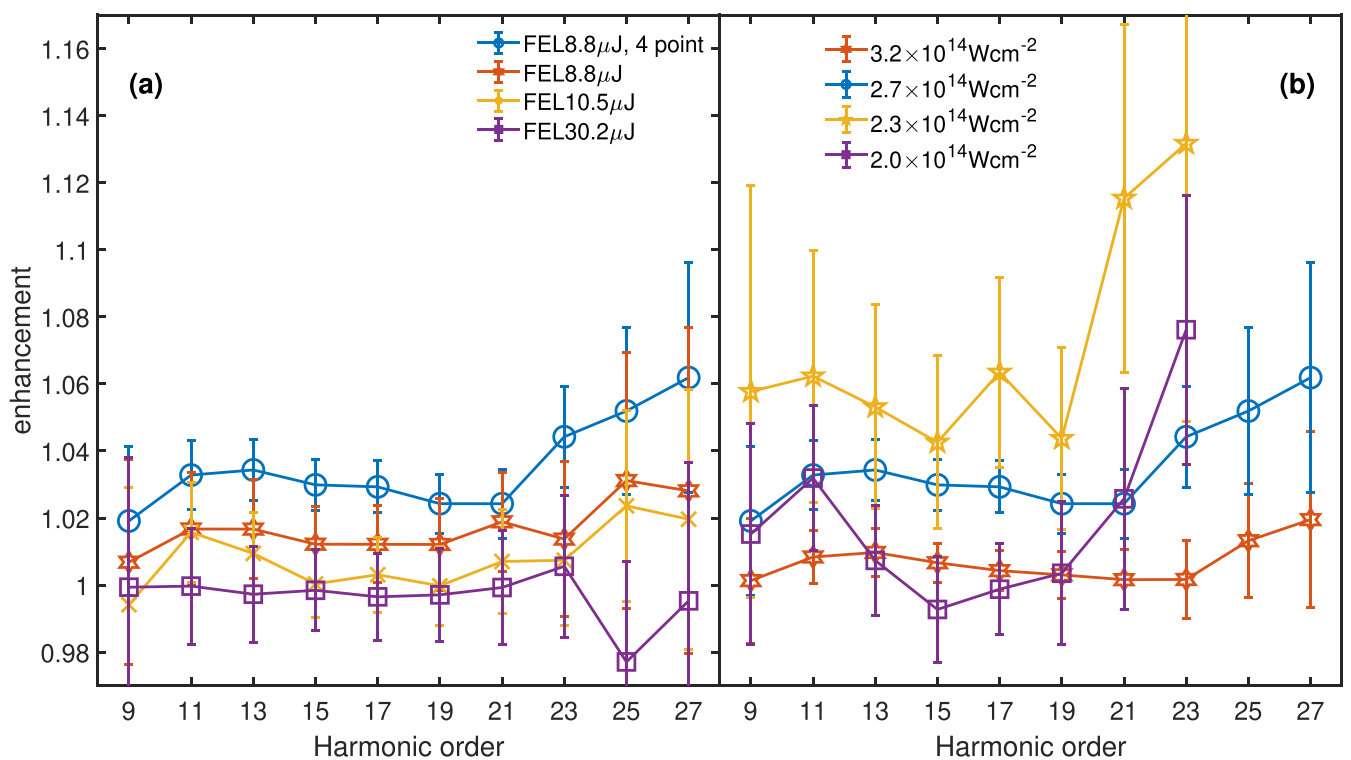

Figure 5. Harmonic yield in $\mathrm{Kr}$ at an FEL photon energy of $82.6 \mathrm{eV}$, integrated over a delay range of -200 to -50 fs and divided by the yield for NIR pulses preceding the FEL pulses by more than $1 \mathrm{ps}$, as a function of harmonic order: (a) plotted for different FEL pulse energies at an NIR intensity of $2.7 \times 10^{14} \mathrm{~W} \mathrm{~cm}^{-2}$, and (b) for different NIR intensities at a fixed FEL pulse energy of $8.8 \mu \mathrm{J}$. The data represented as blue circles are identical for panels (a) and (b).

After finding the FEL pulse energy that resulted in the strongest enhancement of the HHG yield, we then varied the NIR laser power to investigate how the enhancement of the HHG spectrum produced in the presence of both NIR and XUV fields depends on the NIR intensity. In figure 5(b), we plot the enhancement of individual harmonics in krypton gas in the presence of an XUV field with an intensity of
$1.2 \times 10^{12} \mathrm{~W} \mathrm{~cm}^{-2}$ at a photon energy of $82.6 \mathrm{eV}$ as a function of harmonic order for various NIR intensities. At the highest NIR intensity, no enhancement is observed for most harmonic orders, while a clear enhancement appears as the NIR intensity is reduced. At the intensity of $2.3 \times 10^{14} \mathrm{~W} \mathrm{~cm}^{-2}$, we observe an enhancement of $4 \%-6 \%$ in all harmonic orders except for the cutoff harmonics, where the enhancement is 
higher than $10 \%$. This increased enhancement in the harmonics near the cutoff is present in all data sets. At the lowest measured NIR intensity of $2 \times 10^{14} \mathrm{~W} \mathrm{~cm}^{-2}$, we still observe a slight enhancement in the cutoff region, but no enhancement is visible in the plateau region.

Note that for these measurements at different NIR intensities, we only recorded data at four discrete delay values: -1.4 , $-0.1,0.5$, and $2.0 \mathrm{ps}$. The data in figure $5(\mathrm{~b})$ is derived from the ratio of the yields at -0.1 and $-1.4 \mathrm{ps}$. For each of the data points recorded at a fixed delay position, we can use the BAM to more precisely define the delay between the NIR and FEL pulses within the width of the arrival-time jitter, which is approximately $100 \mathrm{fs}$ (FWHM) for this particular data set. When doing such shot-by-shot sorting on the BAM values, especially for the data at $-0.1 \mathrm{ps}$, we observed an additional delay dependence within the data recorded at an NIR intensity of $2.3 \times 10^{14} \mathrm{~W} \mathrm{~cm}^{-2}$. For the data points shown here, only a subset of the data corresponding to the shots in the first half of arrival time range was selected, which showed a larger enhancement than the latter half of the range. This resulted in an enhancement between $5 \%$ and $12 \%$, as shown here, as compared to an enhancement between $3 \%$ and $6 \%$ without the additional sorting. Similar sorting was also attempted on the scans at other NIR intensities but did not show any significant effects, such that the full data sets are shown for the other NIR intensities.

In order to qualitatively explain the observed enhancement, we recall our discussion in section 3 on the phase matching of the HHG process, which influences the response of a macroscopic medium. In particular, the FEL pulse directly influences two terms in the phase-matching equation (3): the plasma-induced phase mismatch, which is mainly affected by the photo and Auger electrons created by the additional ionization of the target gas by the FEL; and the neutral dispersion phase mismatch, which changes as the number of neutral atoms in the medium changes due to the ionization by the FEL. When the FEL and NIR pulses are overlapped in time, the phase matching conditions will thus change dramatically, and it is conceivable that one may, for a given combination of XUV and NIR intensities, end up with an overall phase mismatch that is smaller than in the case where only the NIR pulse is present. Since a quantitative estimate is extremely difficult without precise characterization of the spatial (and possibly also temporal) intensity profiles of both the XUV and the NIR beams, we have not attempted to quantitatively model this effect, but we consider this to be the most likely cause of the observed enhancement in the HHG yield in our experiment. However, given that the photon energy, at which we observe this enhancement, matches the $3 d \rightarrow 4 p$ resonance in singly ionized krypton, it is also possible that the single-atom effects discussed in [20] contribute to the enhanced yield or that neutral or ionic excited states created by the XUV pulse contribute to the HHG process and lead to an enhancement.

An interesting observation in this context is the fact that the enhancement appears to occur only in the leading edge of the FEL pulse for delays between -200 and $-50 \mathrm{fs}$. We believe that this may be due to the interplay between improved phase matching at a specific ionization fraction early on in the FEL pulse, and the depletion of emitters due to ionization, which eventually dominates near the peak of the FEL pulse. As seen in figure 2 and explained above, the harmonic signal starts to drop approximately $100 \mathrm{fs}$ before time zero due to the depletion of emitters. One may argue that the increased harmonic emission due to the improved phase matching should make the signal drop less rapidly than it would due to the depletion effect alone, but since we have no good way to disentangle the two contributions in the experimental data, we observe only the onset of the enhancement before target depletion takes over. Given that the data in figure 5 shows an increasing enhancement for decreasing FEL pulse energy, it might be advantageous in a future study to work at even lower FEL pulse energy to investigate if there is a regime with larger enhancement but negligible depletion. Our hypothesis would be that in that case, the enhancement would also shift closer to time zero.

\section{Summary and conclusions}

We have studied HHG in the presence of intense XUV light and have experimentally investigated various schemes that have been predicted to enhance the harmonic yield, such as resonant excitation of an (inner-shell) electron and creation of a slow photoelectron by the XUV. By performing these experiments at a FEL, extremely high XUV intensities are available, which makes it possible to excite or ionize a much larger fraction of the target atoms than when using a HHG source to produce the XUV/VUV pulses, as it was done in previous studies.

Furthermore, taking advantage of the easier wavelength selectivity of the XUV radiation from an FEL as compared to HHG XUV sources, we studied the dependence on the photon energy and attempted to investigate the effect of resonant inner-shell excitation on the HHG process as well as the effect of creating a photoelectron with low kinetic energy by the XUV pulse, which could then be accelerated by the NIR field.

Overall, we performed studies at 27.2, 15, and $13 \mathrm{~nm}$ in both Ar and Kr. In all targets and at all wavelengths studied, a strong decrease of the HHG yield and a small red shift of the HHG spectrum is observed when the XUV precedes the NIR pulse, which we attribute, based on our model calculations, to a decrease in the number of neutral atoms and an increase in the fraction of ionized target atoms due to the additional ionization induced by the FEL pulse. However, existing propagation models developed to describe the response of a macroscopic medium to a single NIR pulse are not able to reproduce the observed effects quantitatively, suggesting that these models may have to be modified for the present scenario.

We observed a small enhancement of the HHG yield only in $\mathrm{Kr}$ at $15 \mathrm{~nm}$, a wavelength which is in resonance with the $3 d \rightarrow 4 p$ transition in singly ionized krypton. We tentatively identified improved phase matching as the most viable explanation for the observed effect, but also cannot exclude other XUV-assisted HHG mechanisms. More systematic studies than were possible during the short 
beam time that was allocated, and a more quantitative characterization of the XUV and NIR focus profiles and their overlap are needed in order to gather further experimental evidence to confirm or exclude any of the discussed mechanisms.

To the best of our knowledge, this is the first experimental study of HHG in the presence of an intense XUV field from a FEL. Our experiment demonstrates the technical feasibility of XUV-assisted HHG experiments at FEL, which may provide new avenues to investigate correlation-driven electron dynamics as well as novel ways to study and control propagation effects and phase matching in HHG.

\section{Data availability statement}

The data that support the findings of this study are available upon reasonable request from the authors.

\section{Acknowledgments}

This work was supported by the Chemical Sciences, Geosciences, and Biosciences Division, Office of Basic Energy Sciences, Office of Science, U.S. Department of Energy (DOE) under Grant No. DE-FG02-86ER13491. J T was partially supported by the National Science Foundation (NSF) Grant No. PHYS-1753324 to D R. S B acknowledges funding from the Initiative and Networking Fund of the Helmholtz Association (VH-NG-1104). We thank the technical and scientific teams at FLASH for their hospitality and their support during the beam time, in particular Günter Brenner, Daniel Ramm, and Erland Müller, who have been instrumental for the success of this beam time. We acknowledge the Max Planck Society for funding the development and the initial operation of the CAMP end-station within the Max Planck Advanced Study Group at CFEL and for providing this equipment for CAMP@FLASH. The installation of CAMP@FLASH was partially funded by the BMBF Grant Nos. 05K10KT2, 05K13KT2, 05K16KT3 and 05K10KTB from FSP-302. We also acknowledge helpful discussions with Artem Rudenko, Brett Esry, Chii-Dong Lin, and Antonio Picon during the preparation of the beam time proposal and during the interpretation of the data.

\section{ORCID iDs}

Dimitrios Rompotis (D) https://orcid.org/0000-0002-2305092X

Benjamin Erk (D) https://orcid.org/0000-0001-8413-3588 Bastian Manschwetus (D) https://orcid.org/0000-0001-61659560

Rebecca Boll (D) https://orcid.org/0000-0001-6286-4064 Patrik Grychtol (D) https://orcid.org/0000-0002-7042-9334 Carlos Trallero (D) https://orcid.org/0000-0002-9776-8125 Daniel Rolles (D) https://orcid.org/0000-0002-3965-3477

\section{References}

[1] McNeil B W J and Thompson N R 2010 X-ray free-electron lasers Nat. Photon. 4 814-21

[2] Chini M, Zhao K and Chang Z 2014 The generation, characterization and applications of broadband isolated attosecond pulses Nat. Photon. 8 178-86

[3] Popmintchev T et al 2012 Bright coherent ultrahigh harmonics in the $\mathrm{keV} \mathrm{x}$-ray regime from mid-infrared femtosecond lasers Science 336 1287-91

[4] Corkum P B and Krausz F 2007 Attosecond science Nat. Phys. $3381-7$

[5] Bostedt C et al 2016 Linac coherent light source: the first five years Rev. Mod. Phys. 88015007

[6] Young L et al 2018 Roadmap of ultrafast X-ray atomic and molecular physics J. Phys. B: At. Mol. Opt. Phys. 51032003

[7] Appi E et al 2020 A synchronized VUV light source based on high-order harmonic generation at FLASH Sci. Rep. 106867

[8] Bandrauk A D and Shon N H 2002 Attosecond control of ionization and high-order harmonic generation in molecules Phys. Rev. A 66031401

[9] Ishikawa K 2003 Photoemission and ionization of $\mathrm{He}^{+}$under simultaneous irradiation of fundamental laser and high-order harmonic pulses Phys. Rev. Lett. 91043002

[10] Schafer K J, Gaarde M B, Heinrich A, Biegert J and Keller U 2004 Strong field quantum path control using attosecond pulse trains Phys. Rev. Lett. 92023003

[11] Gaarde M B, Schafer K J, Heinrich A, Biegert J and Keller U 2005 Large enhancement of macroscopic yield in attosecond pulse train-assisted harmonic generation Phys. Rev. A 72013411

[12] Schiessl K, Persson E, Scrinzi A and Burgdörfer J 2006 Enhancement of high-order harmonic generation by a two-color field: influence of propagation effects Phys. Rev. A 74053412

[13] Biegert J, Heinrich A, Hauri C, Kornelis W, Schlup P, Anscombe M, Schafer K, Gaarde M and Keller U 2006 Control of high-order harmonic emission using attosecond pulse trains J. Mod. Opt. $\mathbf{5 3} 87-96$

[14] Heinrich A, Kornelis W, Anscombe M, Hauri C, Schlup P, Biegert J and Keller U 2006 Enhanced VUV-assisted high harmonic generation J. Phys. B: At. Mol. Opt. Phys. 39 S275

[15] Gademann G, Kelkensberg F, Siu W K, Johnsson P, Gaarde M B, Schafer K J and Vrakking M J J 2011 Attosecond control of electron-ion recollision in high harmonic generation New J. Phys. 13033002

[16] Brizuela $\mathrm{F}$ et al 2013 Efficient high-order harmonic generation boosted by below-threshold harmonics Sci. Rep. 31410

[17] Brown A and van der Hart H 2016

Extreme-ultraviolet-imitated high-order harmonic generation: driving inner-valence electrons using below-threshold-energy extreme-ultraviolet light Phys. Rev. Lett. 117093201

[18] Tudorovskaya M and Lein M 2014 High-harmonic generation with combined infrared and extreme ultraviolet fields $J$. Mod. Opt. 61 845-50

[19] Leeuwenburgh J, Cooper B, Averbukh V, Marangos J P and Ivanov M 2013 High-order harmonic generation spectroscopy of correlation-driven electron hole dynamics Phys. Rev. Lett. 111123002

[20] Buth C, Kohler M C, Ullrich J and Keitel C 2011 High-order harmonic generation enhanced by XUV light Opt. Lett. 36 3530-2

[21] Buth C 2015 High-order harmonic generation with resonant core excitation by ultraintense x-rays Eur. Phys. J. D 69234 
[22] Buth C, He F, Ullrich J, Keitel C H and Hatsagortsyan K Z 2013 Attosecond pulses at kiloelectronvolt photon energies from high-order-harmonic generation with core electrons Phys. Rev. A $\mathbf{8 8} 033848$

[23] Erk B et al 2018 CAMP@FLASH: an end-station for imaging, electron- and ion-spectroscopy and pump-probe experiments at the FLASH free-electron laser $J$. Synchrotron Radiat. 25 1529-40

[24] Feldhaus J 2010 FLASH-the first soft x-ray free electron laser (FEL) user facility J. Phys. B: At. Mol. Opt. Phys. 43194002

[25] Behrens C, Gerasimova N, Gerth C, Schmidt B, Schneidmiller E A, Serkez S, Wesch S and Yurkov M V 2012 Constraints on photon pulse duration from longitudinal electron beam diagnostics at a soft x-ray free-electron laser Phys. Rev. Spec. Top. Accel. Beams 15030707

[26] Düsterer S et al 2014 Development of experimental techniques for the characterization of ultrashort photon pulses of extreme ultraviolet free-electron lasers Phys. Rev. Spec. Top. Accel. Beams 17120702

[27] Redlin H, Al-Shemmary A, Azima A, Stojanovic N, Tavella F, Will I and Düsterer S 2011 The FLASH pump-probe laser system: setup, characterization and optical beamlines $\mathrm{Nucl}$. Instrum. Methods Phys. Res. A 635 S88-S93

[28] Schulz S et al 2015 Femtosecond all-optical synchronization of an x-ray free-electron laser Nat. Commun. 65938

[29] Savelyev E et al 2017 Jitter-correction for IR/UV-XUV pump-probe experiments at the FLASH free-electron laser New J. Phys. 19043009

[30] Keitel B 2019 private communication

[31] Bellini M, Lyngå C, Tozzi A, Gaarde M B, Hänsch T W, L'Huillier A and Wahlström C-G 1998 Temporal coherence of ultrashort high-order harmonic pulses Phys. Rev. Lett. 81 297-300

[32] Rolles D, Boll R, Erk B, Rompotis D and Manschwetus B 2018 An experimental protocol for femtosecond NIR/UV - XUV pump-probe experiments with free-electron lasers J. Vis. Exp. 140 e57055

[33] Corkum P B 1993 Plasma perspective on strong field multiphoton ionization Phys. Rev. Lett. 711994
[34] Krause J L, Schafer K J and Kulander K C 1992 High-order harmonic generation from atoms and ions in the high intensity regime Phys. Rev. Lett. 683535

[35] Le A, Lucchese R R, Tonzani S, Morishita T and Lin C D 2009 Quantitative rescattering theory for high-order harmonic generation from molecules Phys. Rev. A 80013401

[36] Constant E, Garzella D, Breger P, Mével E, Dorrer C, Le Blanc C, Salin F and Agostini P 1999 Optimizing high harmonic generation in absorbing gases: model and experiment Phys. Rev. Lett. 821668

[37] Lin C-D, Jin C, Le A-T and Wei H 2018 Attosecond and Strong-Field Physics: Principles and Applications (Cambridge: Cambridge University Press)

[38] Durfee C G, Rundquist A R, Backus S, Herne C, Murnane M M and Kapteyn H C 1999 Phase matching of high-order harmonics in hollow waveguides Phys. Rev. Lett. 83 2187-90

[39] Averchi A, Faccio D, Berlasso R, Kolesik M, Moloney J V, Couairon A and Di Trapani P 2008 Phase matching with pulsed Bessel beams for high-order harmonic generation Phys. Rev. A 77021802

[40] Jin C, Le A-T and Lin C D 2011 Medium propagation effects in high-order harmonic generation of $\mathrm{Ar}$ and $\mathrm{N}_{2}$ Phys. Rev. A 83023411

[41] Jin C, Wörner H J, Tosa V, Le A-T, Bertrand J B, Lucchese R R, Corkum P B, Villeneuve D M and Lin C D 2011 Separation of target structure and medium propagation effects in high-harmonic generation J. Phys. B: At. Mol. Opt. Phys. 44095601

[42] Samson J A R and Stolte W C 2002 Precision measurements of the total photoionization cross-sections of $\mathrm{He}, \mathrm{Ne}, \mathrm{Ar}, \mathrm{Kr}$ and Xe J. Electron Spectrosc. Relat. Phenom. 123 265-76

[43] Shin H J, Lee D G, Cha Y H, Hong K H and Nam C H 1999 Generation of nonadiabatic blueshift of high harmonics in an intense femtosecond laser field Phys. Rev. Lett. 832544

[44] Cao W, Laurent G, Jin C, Li H, Wang Z, Lin C, Ben-Itzhak I and Cocke C 2012 Spectral splitting and quantum path study of high-harmonic generation from a semi-infinite gas cell J. Phys. B: At. Mol. Opt. Phys. 45074013

[45] Popmintchev D et al 2015 Ultraviolet surprise: efficient soft $\mathrm{x}$-ray high-harmonic generation in multiply ionized plasmas Science 350 1225-31 